\title{
Role of leaf mines in host location and pupation in Diglyphus isaea (Hymenoptera: Eulophidae)
}

\author{
De Yu ZOU ${ }^{1,2}$, Hong YIN CHEN ${ }^{1,2 *}$, Li SHENG ZHANG ${ }^{1,2}$ \\ ${ }^{1}$ Key Laboratory of Integrated Pest Management in Crops, Ministry of Agriculture, Institute of Plant Protection, Chinese Academy \\ of Agricultural Sciences, 12 Zhongguancun Nandajie, Haidian, Beijing 100081, China \\ ${ }^{2}$ Sino-American Biological Control Laboratory (SABCL), USDA-ARS, 12 Zhongguancun Nandajie, Haidian, Beijing 100081, \\ China; e-mails: deyuzou@gmail.com; chen.hongyin@gmail.com; zhangleesheng@163.com
}

Key words. Hymenoptera, Eulophidae, Diglyphus isaea, Diptera, Agromyzidae, Liriomyza sativae, leaf miner, artificial mine, host searching ability, pupation

\begin{abstract}
Diglyphus isaea Walker (Hymenoptera: Eulophidae) is an important ectoparasitic wasp of many leaf miners. Ability of $D$. isaea to find hosts placed in artificial mines and for its larvae to pupate when the larva is not in a mine was studied. Artificial mines consisted of slits cut in index card sandwiched between two cover slips. Almost $80 \%$ of the neonate larvae of $D$. isaea located host larvae in artificial mines compared to only $50 \%$ of those not in a mine. Mature larvae removed from mines did not construct normal pupal chambers. Nonetheless, they pupated and emerged successfully. Larvae of Liriomyza sativae Blanchard (Diptera: Agromyzidae) in mines are more likely to be attacked than those not in mines. Moreover, when close to a host larva this parasitoid can use vibrational cues to locate the larva in a mine. In addition, this parasitoid also did not always use volatile and gustatory cues for short range location of hosts. However, adult females of $D$. isaea more quickly located $L$. sativae larvae in the presence of the odour of juice extracted from an uninfested host plant. We conclude by proposing that the host mine is the medium by which the vibrations generated by the host larva are transmitted, which are probably the most important cue used by female $D$. isaea searching for hosts. That is this parasitoid first perceives mines not host larvae. These results will be helpful for developing techniques for the mass rearing of $D$. isaea in the future.
\end{abstract}

\section{INTRODUCTION}

The pests, Liriomyza sativae Blanchard, 1938 (Diptera: Agromyzidae) and Liriomyza trifolii Burgess, 1880 (Diptera: Agromyzidae) invaded China in 1993 and 2005, respectively. Leaf miners in the genus Liriomyza (Diptera: Agromyzidae) are serious pests of agricultural crops, especially vegetables in greenhouses (Cheng et al., 2004; Lei et al., 2007). Diglyphus isaea Walker, 1838 (Hymenoptera: Eulophidae) has received a lot of attention because it has been used effectively to reduce the abundance of agromyzid leaf miners (Hendrickson \& Barth, 1979; Heinz et al., 1988, 1993; LaSalle \& Parrella, 1991; Sher et al., 1996; Zhu et al., 2000).

D. isaea is an ectoparasitic, synovigenic, idiobiont wasp capable of developing on at least 18 different agromyzid species mainly associated with herbaceous plants. It has also been recorded parasitizing Lyonetia clerckella Linnaeus, 1758 (Lepidoptera: Lyonetiidae) larvae (Bouček \& Askew, 1968). Female D. isaea attack the second and third instar larvae, which they immobilize by stinging before host-feeding or parasitizing. When a female host-feeds it stings the host many times and feeds upon the fluid that exudes from the wounds. Kim et al. (2007) studied the ecological and biological characteristics of $D$. isaea and revealed that at $25^{\circ} \mathrm{C}$ the egg, larval, pupal and adult stages take 1.3, 4.5, 6.3 and 25.1 days, respectively, to complete their development. A female can lay 305.3 eggs and parasitize/kill 962.2 L. trifolii throughout its lifetime.

Much of the biology of $D$. isaea, however, remains to be studied (Minkenberg, 1989; Cheah \& Coaker, 1992). D. isaea locate host larvae using volatile signals released by infested plants (Valérie et al., 1996). All stages, except adult $D$. isaea, live in host mines. The advantages of feeding on a host larva within a leaf mine include: a lower incidence of disease, a microenvironment with lower evaporative demand and therefore protection from desiccation, protection afforded by the leaf-mine epidermis from the direct and indirect effects of UV radiation and avoidance of attack by natural enemies, resulting in higher survival (Connor \& Taverner, 1997). Host mines, however, may have other long-term functions in coevolution. Furthermore, because there are few, if any, studies on parasitoids presented with hosts in artificial mines or removed from mines, we carried out research on the response of the ectoparasitoid $D$. isaea to the leaf miner L. sativae in artificial and natural mines under laboratory conditions. The objectives of this study were to investigate whether $D$. isaea adults can find host larvae that are not in mines or in the absence of volatile cues from plants and the role of host mines in locating a host. In this study all stages of $D$. isaea were investigated and the results were discussed in detail. Moreover, there are no studies on rearing $D$. isaea on larvae other than those

\footnotetext{
* Corresponding author.
} 
from which it is recorded in the field. Therefore, we selected the house fly, Musca domestica vicina Macquart, 1851 (Diptera: Muscidae), as an artificial host. As it is easy to rear this parasitoid in vitro the suitability of house fly larvae as a host for $D$. isaea was also investigated using artificial leaves.

\section{MATERIAL AND METHODS}

\section{Rearing}

The experiments were conducted at $27 \pm 1{ }^{\circ} \mathrm{C}$ and $65-69 \%$ relative humidity using $L$. sativae as the host and English red kidney bean (Phaseolus vulgaris L.) as the host plant of the leafminer. D. isaea was cultured on hosts in English red kidney bean leaves. The L. sativae and D. isaea were obtained from cultures maintained in our laboratory, the Sino-American Biological Control Laboratory. Adult house flies, obtained from the Chinese Academy of Agricultural Sciences in Beijing, were fed on brown sugar and water, and provided with bran moistened with milk for egg laying and as a diet for the house fly larvae. The photoperiod used was $14 \mathrm{~L}: 10 \mathrm{D}$.

\section{Ability of neonate larvae of $D$. isaea to locate hosts that are not in mines and those placed in artificial mines}

Just as a blind person uses a cane as a guide, the host mine may be an important object of reference for blind larvae of $D$. isaea searching for a host. In the absence of a mine, which serves to confine a neonate parasitoid to the close proximity of its host, the parasitoid might be less successful in finding the host. When a parasitoid comes to the edge of a mine, it must turn around or take other corrective action if it is to find the host and in the absence of this constraint it might simply wander off. Therefore, tests of the ability of neonate larvae of $D$. isaea to search for hosts were conducted to determine if they need to be in a mine in order to find immobilized host larvae. Immobilized host larvae were used because females immobilize their hosts before ovipositing. In this experiment, neonate larvae were obtained by exposing host plants infested with second and third instar larvae of $L$. sativae to females of $D$. isaea for $24 \mathrm{~h}$. The eggs of $D$. isaea and their host larvae were then selected at random from the host plants and transferred onto \#5 qualitative filter paper moistened with distilled water and arranged as they were in the natural host mines in order to present the parasite larva with a realistic test of its ability to find its host in artificial host mines or in an open arena. Some eggs were deposited next to host larvae. But most eggs were separated from and at an angle or parallel to the host larva. The distance between host larvae and wasp eggs was about the length of one or two eggs of $D$. isaea. The qualitative filter paper with the egg of $D$. isaea and its host larva was then placed in a covered, $9 \mathrm{~cm}$ diameter

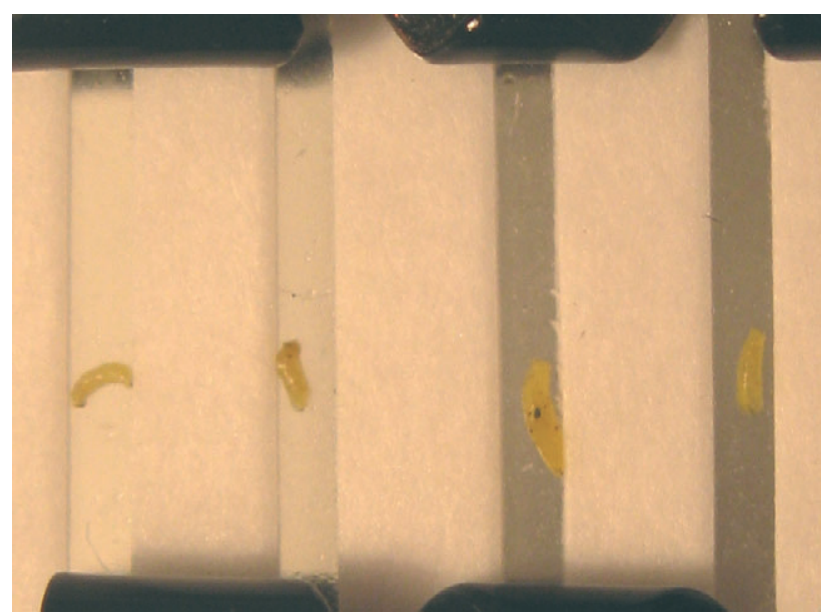

Fig. 1. Artificial mine system consisting of slits cut in an index card with the larvae of Liriomyza sativae Blanchard in situ.

Petri dish. Distilled water was added to the filter paper when required to keep it moist. There was one host larva and one egg per Petri dish and this treatment was replicated 30 times. Another $30 \mathrm{D}$. isaea eggs and host larvae were transferred to artificial mines. The artificial mine system consisted of four slits $(1.7 \mathrm{~cm}$ long $\times 0.1 \mathrm{~cm}$ wide) cut parallel to each other in a piece of blank index card $(2 \mathrm{~cm}$ long $\times 2 \mathrm{~cm}$ wide, moistened with distilled water). This piece of card was placed on a cover slip (2 $\mathrm{cm}$ long $\times 2 \mathrm{~cm}$ wide). An egg and its immobilized host larva were positioned as described previously in the center of each of the artificial mines (slits) and then another cover slip ( $2 \mathrm{~cm}$ long $\times 2 \mathrm{~cm}$ wide) was placed over the index card so that the open end of each mine vented into the atmosphere. Each of the four mines contained an egg and an immobilized host larva. The artificial mine system, held together with two clips, was placed on \#5 qualitative filter paper moistened with distilled water in a covered, $9 \mathrm{~cm}$ diameter Petri dish. Distilled water was added to the filter paper as required to prevent it from drying out. Whether a neonate larvae of $D$. isaea had found its host was monitored at $08 \mathrm{~h} 00$ and $14 \mathrm{~h} \mathrm{00,} \mathrm{every} \mathrm{day,} \mathrm{for} \mathrm{four} \mathrm{days.} \mathrm{This}$ treatment was replicated three times. The artificial mine system is shown in Fig.1.

\section{Pupation and emergence of $D$. isaea not in a mine and those placed in artificial mines}

It was very difficult to observe the pupal chambers that are constructed by mature larvae of $D$. isaea between the upper and lower epidermis of leaves. Therefore, artificial mines were used
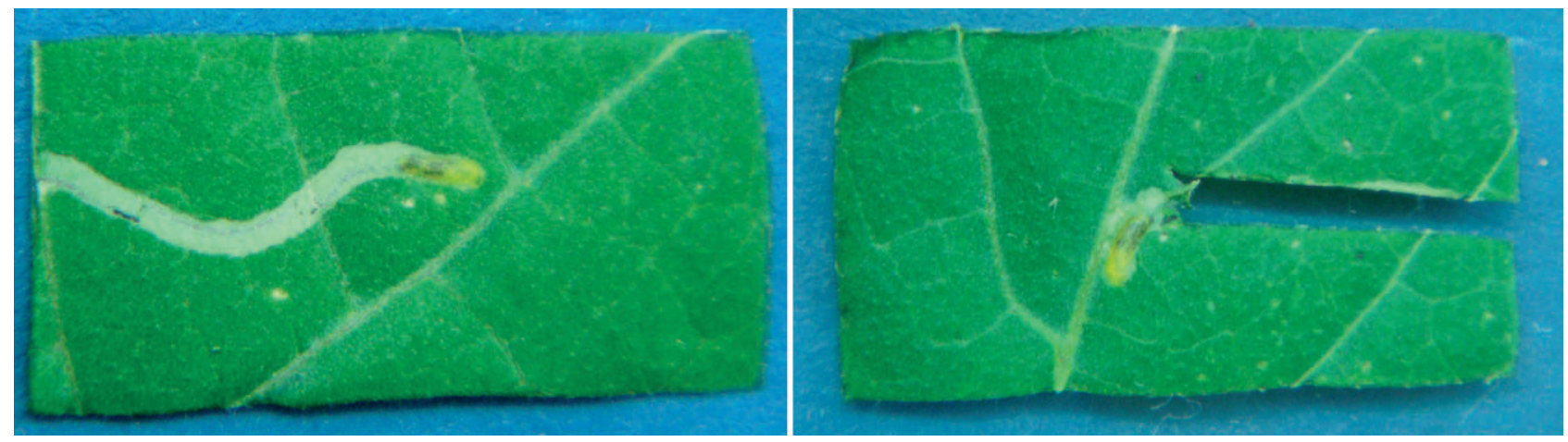

Fig. 2. Pieces of leaf with a complete and trimmed mine of Liriomyza sativae Blanchard, respectively. 
TABLE 1. List of different treatments used to analyze the influence of vibrational signals and host plant odours on the numbers of larvae of different hosts that were attacked by Diglyphus isaea.

\begin{tabular}{clll}
\hline Treatments & Larvae & Mines in artificial leaves & $9 \mathrm{~cm}$ Petri dish \\
\hline 1 & Healthy L. sativae & $\begin{array}{l}\text { Smeared with leaf juice } \\
\text { extracted from a healthy host plant }\end{array}$ & Without leaf balls \\
2 & Healthy L. sativae & Not smeared with leaf juice & Contains 5 leaf balls \\
3 & Healthy L. sativae & Not smeared with leaf juice & Without leaf balls \\
4 & Healthy Musca domestica & $\begin{array}{l}\text { Smeared with leaf juice } \\
\text { extracted from a healthy host plant }\end{array}$ & Contains 5 leaf balls \\
& $\begin{array}{l}\text { Dead L. sativae killed by dipping them } \\
\text { into hot water for 1 min }\end{array}$ & Not smeared with leaf juice & Contains 5 leaf balls \\
\hline
\end{tabular}

to assess whether mature larvae of $D$. isaea need mines in which to pupate and emerge. Forceps were used to remove the leaf epidermis from above host mines under a dissecting microscope. One mature larva of $D$. isaea, after completion of host-feeding but before it constructed a pupal chamber, was transferred onto filter paper regularly moistened with distilled water in a covered, $9 \mathrm{~cm}$ diameter Petri dish. There was one larva per Petri dish and a total of 30 mature larvae of $D$. isaea were used in this experiment. Another 30 mature larvae of $D$. isaea were transferred to artificial mines. A total of 60 mature larvae of $D$. isaea were used to investigate the characteristics of the pupal chambers, pupal developmental period and adult emergence, which were recorded at $08 \mathrm{~h} 00$ and $20 \mathrm{~h} 00$ every day for six days. This treatment was replicated three times.

\section{Role of host mines in determining the ability of female $D$. isaea to locate and parasitize the larvae of leaf miners}

Host mines are used by females of $D$. isaea to locate host larvae. In this experiment, the role of host mines in determining the ability of female $D$. isaea to locate a host was tested. A second or third instar larva of $L$. sativae was placed in the center of a piece of excised leaf $(2 \mathrm{~cm}$ long $\times 1 \mathrm{~cm}$ wide $)$ of English red kidney bean. This was replicated 10 times. The natural mines of L. sativae were cut using scissors from five excised leaves (larvae were verified as still alive if their mouth hooks moved vigorously) in such a way that only the portion where the larva was located was included, as shown in Fig. 2. The natural mines of $L$. sativae were not trimmed from another five excised leaves. Then these ten pieces of excised leaves were randomly placed in a $9 \mathrm{~cm}$ Petri dish along with 10 mature and experienced $D$. isaea females for $0.5 \mathrm{~h}$. This was replicated 10 times. A total of $100 \mathrm{D}$. isaea females were used in this test. The number of parasitoid attacks was recorded.

Role of gustatory cues associated with mines in determining the ability of female $D$. isaea to locate and parasitize the larvae of leaf miners

Female D. isaea search along host mines for host larvae. Artificial leaves were used to test whether a gustatory stimulus on the surface of a leaf is used to locate host larvae. Host mines were dissected and early third instar larvae of $L$. sativae were removed and each placed on a cover slip $(2 \mathrm{~cm}$ long $\times 2 \mathrm{~cm}$ wide). Then to form an artificial leaf the cover slip was wrapped in a thin layer of parafilm. Edges of the cover slip were held between fingers to prevent the larvae from escaping. The surfaces of five artificial leaves were then smeared with juice extracted from healthy host plants. Another five artificial leaves containing host larvae were rubbed with the upper epidermis of normal mines. These 10 artificial leaves, arranged so that a leaf smeared with juice from an uninfected plant was placed next to one rubbed with the epidermis from above a mine and vice versa, were placed in a $9 \mathrm{~cm}$ Petri dish with 10 mature and expe- rienced $D$. isaea females for $3 \mathrm{~h}$. Then the total number of hosts attacked, which included the number of hosts upon which eggs were deposited and attacks by adult females that did not result in oviposition, and number of eggs per host laid by $D$. isaea, were recorded. If host larvae escaped from artificial leaves, a replacement was tested. This sequence was replicated 10 times. A total of $100 \mathrm{D}$. isaea females were used (10 for each replicate) in this test.

Role of host plant odours and vibrations in determining the ability of female $D$. isaea to locate and parasitize the larvae of $L$. sativae and M. domestica

Because female $D$. isaea did not recognize host larvae isolated from mines, we tested the ability of female $D$. isaea to locate and parasitize non-host larvae (1) in the absence of cues associated with artificial mines or host plant odours, and (2) with and without any chemical signal. In addition, we tested whether female $D$. isaea were able to locate host larvae in host mines in the absence of vibrations caused by host movement within the mines. Artificial mines in artificial leaves, which simulate natural leaves, were used. In each treatment five larvae were placed in mines in artificial leaves as shown in the Table 1. The second to third instar and second instar larvae of L. sativae and $M$. domestica, respectively, were used in the different treatments. Pieces $(2 \mathrm{~cm}$ long $\times 1 \mathrm{~cm}$ wide $)$ of leaves from uninfested host plants were converted into balls by rubbing them between two fingers, which resulted in the release of more odour. These balls were placed at the edges of a $9 \mathrm{~cm}$ Petri dish and provided a source of host plant odour. Then five mature and experienced female $D$. isaea were introduced for $3 \mathrm{~h}$. Every dish contained five artificial leaves. The number of hosts on which eggs were laid and number of females that attacked larvae but did not lay an egg, which is the total number of hosts attacked, and eggs per host deposited by $D$. isaea, were recorded. In treatment $5 \mathrm{D}$. isaea were offered dead larvae to assess if the parasitoid can locate dead larvae that lack the vibrational cues associated with moving larvae. Larvae were killed by putting them in hot water for $1 \mathrm{~min}$. After three hours, larvae were checked to see whether they were parasitized (larvae were verified as attacked by their immobilization in the live treatment, because $D$. isaea female stings to immobilize the larva before hostfeeding or parasitization; for larvae killed by immersing them in hot water, by the presence of exudate from the sting). If some of the larvae escaped from artificial leaves, another group was tested. Fifty larvae were used in each treatment.

\section{Statistical analysis}

Chi-squared tests of $2 \times 2$ tables were used to compare the numbers of neonate larvae of $D$. isaea that found hosts, dead eggs recorded in artificial mines and in the absence of mines. ANOVA and Fisher's LSD test $(\alpha=0.05)$ was used to compare variables in Experiment 5. Two sample t-tests for means were 
used to compare the development times of $D$. isaea pupae in the presence and absence of artificial mines. Two sample paired t-tests for means were used to compare the numbers of larvae attacked in pieces of leaf with complete mines $v s$. mines trimmed to include only the portion with the larva and the numbers of larvae attacked in artificial leaves smeared with leaf juice extracted from healthy host plants $v s$. those rubbed with the upper epidermis of host mines. SAS (9.0) was used for statistical analysis.

\section{RESULTS}

\section{Ability of neonate larvae of $D$. isaea to locate hosts that are not in mines and those placed in artificial mines}

Almost all the neonate larvae of $D$. isaea in artificial mines located host larvae, whereas significantly fewer of those not in mines did so $\left(\mathrm{X}^{2}=16.632 ; P<0.01\right)$. Neonates of $D$. isaea that failed to find host larvae starved to death. The numbers of dead eggs recorded in the two treatments were not significantly different $\left(\mathrm{X}^{2}=0.247 ; P\right.$ $>0.05)$.

\section{Pupation and emergence of $D$. isaea not in a mine and those placed in artificial mines}

The larvae of $D$. isaea did not need an artificial mine in order to pupate and successfully emerge as adults, but none of them constructed normal pupal chambers. Different kinds of faecal arrangements were recorded (Table 2 ). Of the 63 larvae of $D$. isaea that did not develop in artificial mines, the pupal chamber of each of them had only one faecal pillar, which varied in shape, from pillar like to granule like, cup shaped and even sheet shaped. Only two larvae out of the total of 90 that developed in artificial mines did not construct pupal chambers. In artificial mines, the mature larvae crawled backwards as they constructed their pupal chambers and deposited faecal pillars to the left and right. The pupal chamber was then constructed between the faecal pillars. Thus for the construction of a normal pupal chamber the walls and a roof of a mine were necessary. Table 2 shows that, there were no significant differences between the development times of $D$. isaea that pupated in artificial mines and those that did not $(\mathrm{t}=1.950 ; \mathrm{df}=178 ; P>0.05)$.

\section{Role of host mines in determining the ability of female $D$. isaea to locate and parasitize the larvae of leaf miners}

Almost twice as many L. sativae larvae $(4.2 \pm 0.3$, mean $\pm \mathrm{SE}$ ) in intact natural leaf mines were attacked by

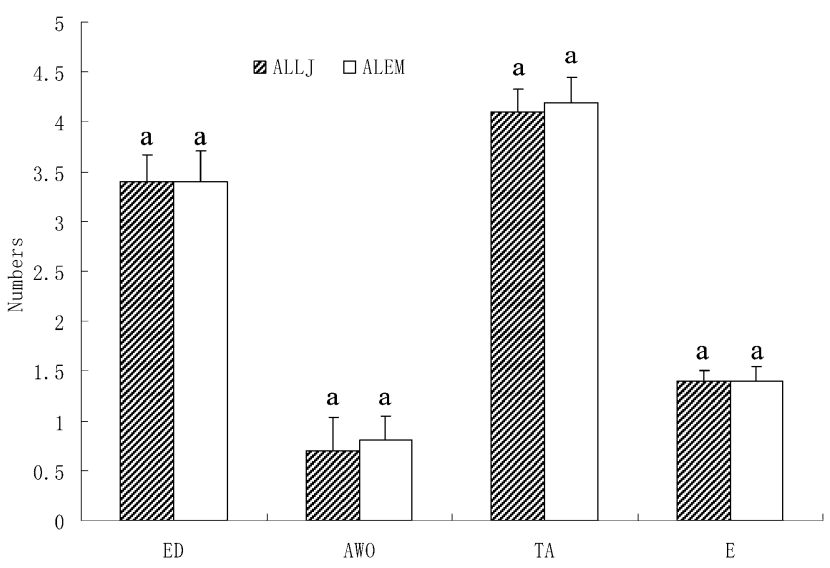

Fig. 3. Effect of smearing artificial leaves with leaf juice or rubbing them with epidermis of mines on the numbers of Liriomyza sativae attacked by Diglyphus isaea. Data are presented as mean \pm SE. ALLJ: Artificial leaves smeared with leaf juice of healthy host plant. ALEM: Artificial leaves rubbed with the upper epidermis of mines. ED: Hosts on which eggs were deposited. AWO: Hosts attacked but not parasitized. TA: Total number of hosts attacked. E: Mean number of eggs laid on each host. Values with the same letter do not differ significantly $(P>$ 0.05 , t-test).

$D$. isaea than of those in trimmed mines $(2.1 \pm 0.3$, mean $\pm \mathrm{SE})$. There were significant differences between the two treatments $(\mathrm{t}=7.584 ; \mathrm{df}=9 ; P<0.01)$. The host larvae produced $1-1.5 \mathrm{~mm}$ of mine by feeding in given time period.

Role of gustatory cues associated with mines in determining the ability of female $D$. isaea to locate and parasitize the larvae of leaf miners

Host larvae in mines in artificial leaves were successfully parasitized even though the textures of artificial and natural leaves were completely different (Fig. 3). The mean number of host larvae attacked in artificial leaves smeared with juice extracted from the leaves of uninfested host plants was $4.1 \pm 0.2($ mean \pm SE) and of those rubbed with the upper epidermis of mines was $4.2 \pm 0.3$ (mean $\pm \mathrm{SE}$ ). Analysis of the data revealed that the incidence of parasitization in both cases were not statistically different $(\mathrm{t}=-0.318$; $\mathrm{df}=9 ; P>0.05)$. Therefore, it is likely that this parasitoid does not use a specific gustatory cue associated with the epidermis of host mines when searching for the larvae of its host.

TABLE 2. Characteristics of the pupal chambers that were constructed by the larvae of Diglyphus isaea in artificial mines and of those mature larvae that were dissected out of mines and kept in Petri dishes, and the development times (d) of the pupae in these two treatments.

\begin{tabular}{|c|c|c|c|c|c|c|c|c|c|c|}
\hline & \multicolumn{9}{|c|}{ Number of faecal pillars per pupal chamber } & \multirow{2}{*}{$\begin{array}{c}\text { Development time of } \\
\text { pupa }(d) * *(\text { mean } \pm S E)\end{array}$} \\
\hline & 0 & 1 & 2 & 3 & 4 & 5 & $6^{*}$ & 8 & 10 & \\
\hline Pupae constructed in artificial mines $(n=90)$ & 0 & 0 & 3 & 6 & 28 & 14 & 21 & 14 & 4 & $4.48 \pm 0.05$ \\
\hline Pupae constructed in Petri dishes $(\mathrm{n}=90)$ & 2 & 63 & 7 & 5 & 6 & 4 & $3^{\#}$ & 0 & 0 & $4.33 \pm 0.05$ \\
\hline
\end{tabular}

* Six faecal pillars were recorded only in one pupal chamber. ${ }^{*}$ Three of the larvae kept in Petri dishes constructed a pupal chamber with six faecal pillars. ** Two sample t-test for means: $\mathrm{t}=1.950, \mathrm{df}=178, P>0.05$. 


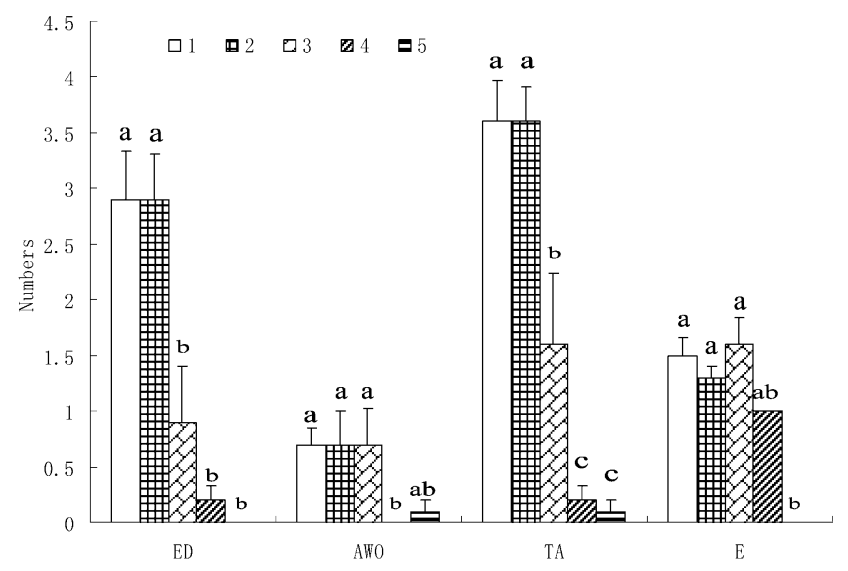

Fig. 4. Influence of vibrational cues and host plant odours on the numbers of different hosts attacked by Diglyphus isaea. Data are presented as mean \pm SE. Treatments 1, 2, 3, 4 and 5 are shown in Table 1. ED: Hosts on which eggs were deposited, AWO: Hosts attacked without oviposition, TA: Total hosts attacked, E: Mean number of eggs laid on each host. Different letters above the bars for each of the treatments indicate significant differences between bars (ANOVA, $P<0.05$; Fisher's LSD, $\alpha=0.05$ ).

\section{Role of host plant odours and vibrations in determining the ability of female $D$. isaea to locate and parasitize the larvae of $L$. sativae and $M$. domestica}

The mean number of $L$. sativae larvae attacked by $D$. isaea in treatment 1 was the same as that in treatment 2 indicating that $D$. isaea did not use volatile odours to locate a host. More than twice as many of the larvae of $L$. sativae in treatment 2 were attacked by $D$. isaea than in treatment 3 , which indicates that $D$. isaea adults were able to locate hosts without the help of volatile chemical cues, but that they may stimulate $D$. isaea to search for host larvae more intensively. Far fewer $M$. domestica larvae in treatment 4 were attacked by $D$. isaea than $L$. sativae larvae in treatment 2 , which probably indicates that the vibrations associated with larvae of $L$. sativae and $M$. domestica differed. Far more $L$. sativae larvae in treatment 2 were attacked by $D$. isaea than in treatment 5 , which indicates that the vibrations may enable this parasitoid to locate a host at close range. The details are presented in Fig. 4.

\section{DISCUSSION}

Research on the effects of temperature on the life history of $D$. isaea indicates that most deaths occur during the egg-larval stage (Minkenberg, 1989). According to our studies, almost half of the neonate larvae of $D$. isaea placed in covered Petri dishes, failed to find a host larva compared to those confined with a host larva in artificial mines. Larvae of $D$. isaea that failed to locate a host after hatching did not survive. Female $D$. isaea preferred to lay eggs on host larvae, but frequently were prevented from doing so by host mines, especially when the mine was not a single chamber but a network of crisscrossing mines or a few interlacing small mines. In that case, a female of $D$. isaea was not always able to locate a host larva, so eggs might be placed far from the host. Junctions between mines might interfere with the ability of neonate larvae of $D$. isaea to find hosts. It appeared that neonate larvae of $D$. isaea search at random to find hosts, therefore it's important that the egg is placed as close to a host as possible. By limiting the area that they have to search host mines make it easier for the parasitoid larva to locate its host.

Heinz \& Parrella (1989) conducted studies on the attack behaviour and host size selection by Diglyphus begini Ashmead, 1904 (Hymenoptera: Eulophidae), a congener of $D$. isaea. They show that larvae of $D$. begini can complete their development in artificial mines very similar to those used in this study. Similarly, Ode \& Heinz (2002) also report that $D$. isaea can complete its development in artificial mines. But these two studies do not describe how neonate larvae find their hosts or construct pupal chambers. The mature larvae of $D$. isaea crawl backwards along mines after feeding on their hosts and construct pupal chambers between pillars of faeces. Larvae press their tail against the roof of the mine and deposit frass, which is stacked to form pillars that extend from the floor to the roof of the mine. The pillars of faeces are always distributed in pairs one each side along the complete length of a larva. These pillars are unstable and collapse if there is no roof to support them. We speculate that the action of pressing their tails against the roof of a mine when building the faecal pillars assists in the process of excretion. If they do not have a roof or wall to press against then they find it difficult to excrete and as a result they produce fewer faecal pillars. We expected that the pupal chamber would have other numbers of faecal pillars in artificial mines, such as seven, nine, 11 and 12, with increased experimental times. Viggiani (1964) describe the peculiar behaviour of the larvae of Kratochviliana and Diglyphus, which also parasitize leaf-mining insects. The last stage larvae of these parasitoids, just prior to pupation, deposit faeces in the form of pellets around their body in order to support the mine walls and provide space for pupation. This type of behaviour is cited as one of the most interesting examples of highly developed host adaptation.

D. isaea searched along mines moving its antennae across the width of the mine, irrespective of whether a host larva was present or not. Exploratory probes into the mine were mostly made close to the host (Cheah \& Coaker, 1992). Interestingly in our studies, more $L$. sativae larvae in normal host mines in excised leaves were attacked by $D$. isaea than in mines that were trimmed except for the portion containing the larva, suggesting that the host mine is used by $D$. isaea as an important cue for locating hosts. It is likely that the mine reduces the area that needs to be search and the likelihood that a potential host will escape detection. Cheah \& Coaker (1992) report that at a close range, visual stimuli are unnecessary for oviposition and host-feeding. In addition, our observation that some female $D$. isaea located a host but laid an egg in a nearby host mine, also revealed that visual cues are not used in host-location. Our data 
also revealed that the mean numbers of host larvae parasitized by $D$. isaea in artificial leaves either smeared with juice extracted from un-infested host plants or rubbed with the leaf epidermis from above a host mine were not distinctly different, which indicates that no specific gustatory stimulus present on the epidermis of host mines was utilized by $D$. isaea for locating hosts.

Some previous studies show that female $D$. isaea are strongly attracted to the volatile signals released by the plant-host complex (Finidori-Logli et al., 1996; Valérie et al., 1996) and the odours from physically damaged nonhost plant leaves strongly increase the EAG (electroantennogram) responses of D. isaea (Zhao \& Kang, 2002). Neither naive nor experienced females show any response to the frass of third-instar host larvae. Only four out of a total of 37 females tested showed a positive response to frass and leaf juice, but in each of these cases, as previously recorded, leaf juice, but not frass, stimulated intensive searching behaviour (Cheah \& Coaker, 1992). Our studies suggest that $D$. isaea adults were able to locate hosts without the help of volatile chemical cues, although when close to a host they may stimulate $D$. isaea to search more actively for host larvae. Long-distance signals probably convey only information about the host's habitat or host plant, while mechanical signals perceived over a short range probably provide more reliable and direct information about the availability of hosts and where they can be found, compared with semiochemical substances emanating from infested plants or the host's faeces or other secretions. A parasitoid probably uses a continuous and dynamic flow of information when searching for a host.

The putative role of vibrational signals in host location by parasitoids is supported by many studies (Meyhöfer et al., 1994; Meyhöfer \& Casas, 1999; Fischer et al., 2001). Similarly in our study most female $D$. isaea failed to locate immobilized hosts. But which kind of vibration is used in host-location, substrate vibrations or air particle movements, remains to be investigated. In our unpublished studies trichoid sensilla mechanoreceptors on the tarsi of female $D$. isaea were recorded reacting to vibrations associated with the movement of a L. sativae larva, which probably also supports the possible role of substrate vibrations. Whether there are sensilla on the antennae or other organs of $D$. isaea that react to air particle movements also needs to be studied. Odours, substrate vibrations, air particle movements or texture are just some of the initial cues that $D$. isaea may use to locate a host. Once perceived it mostly searches mines for the larvae of its host. The more cues they perceive the less time they spend searching. Live host larvae isolated from mines are ignored by female $D$. isaea (Cheah \& Coaker, 1992; Finidori-Logli et al., 1996). This is also the case in Elasmus sp. (Hymenoptera: Eulophidae) and Apechthis rufata Gmelin, 1790 (Hymenoptera: Ichneumonidae) (He, 1980). D. isaea female adults do not even probe and feed on larvae isolated from mines. $D$. isaea larvae have experience of host larvae in mines but do not locate them visually. So adults do not respond to host larvae that are not in a mine even though they have a memory. In our experiment, $D$. isaea only laid an egg on one house fly larva in an artificial leaf smeared with juice extracted from the leaf of a healthy host plant. Perhaps female adults of $D$. isaea can discriminate between hosts and non-hosts in artificial leaves, before probing, using vibrational cues. The study of Coaker \& Cheah (1993) suggest that the oviposition preference of $D$. isaea is determined only by the type of plant in which the host is located. It is suggested that insectivorous parasitoids evolved from stem borers, which encountered other species of larvae in their mines and consumed them. We hypothesize that the host mine is probably a medium for transmitting vibrations that are an important cue in hostlocation and a female $D$. isaea first perceives mines not host larvae. For female $D$. isaea their offspring's food are in the mines (under the leaf epidermis). That is, they recognize the host larvae and mines as a whole system.

ACKNOWLEDGEMENTS. We wish to thank K.A. Hoelmer, R.W. Fuester (Agricultural Research Service, USDA) and N.H. Khuhro (Nuclear Institute of Agriculture Tandojam, Pakistan) for improving the English manuscript. We also extend our thanks to all reviewers for their useful comments. This research was partly supported by Special Fund for Agro-scientific Research in the Public Interest (no. 201103002), Project of International Cooperation of Agriculture of China (no. 2011-G4) and Sino-America Biocontrol International Cooperation Program (no. 58-4001-4-053).

\section{REFERENCES}

Boučex Z. \& Askew R.R. 1968: Hymenoptera, Chalcidoidea, Palearctic Eulophidae (excl. Tetrastrichinae). In Delucchi V. \& Remaudière G. (eds): Index of Entomophagous Insects. Le François, Paris, 254 pp.

Cheah C.A. \& Coaker T.H. 1992: Host finding and discrimination in Diglyphus isaea, a parasitoid of the chrysanthemum leaf miner, Chromatomyia syngenesiae. Biocontrol Sci. Tech. 2: $109-118$.

Cheng W.N., Li X.L., Wu J.X., Li J.J. \& Li Y.P. 2004: Progressive study on occurrence and control of Liriomyza sativae Blanchard. J. Northwest Agric. Forest Univ. (Nat. Sci.) 32: 78-82 [in Chinese].

COAKer T.H. \& Cheah C.A. 1993: Conditioning as a factor in parasitoid host plant preference. Biocontrol Sci. Tech. 3: 277-283.

Connor E.F. \& TAVerner M.P. 1997: The evolution and adaptive significance of the leaf-mining habit. Oikos 79: 6-25.

Finidori-Logli V., Bagnères A.-G. \& Clément J.-L. 1996: Role of plant volatiles in the search for a host by parasitoid Diglyphus isaea (Hymenoptera: Eulophidae). J. Chem. Ecol. 22: 541-558.

Fischer S., SAmietz J., WÄcKerS F.L. \& Dorn S. 2001: Interaction of vibrational and visual cues in parasitoid host location. J. Comp. Physiol. (A) 187: 785-791.

HE J.H. 1980: How do parasitoids find their hosts. Entomol. Knowl. 2: 83-85 [in Chinese].

Heinz K.M. \& Parrella M.P. 1989: Attack behavior and host size selection by Diglyphus begini on Liriomyza trifolii in chrysanthemum. Entomol. Exp. Appl. 53: 147-156.

Heinz K.M., Newman J.P. \& Parrella M.P. 1988: Biological control of leafminers on greenhouse marigolds. Calif. Agric. 42: $10-12$. 
Heinz K.M., Nunney L. \& Parrella M.P. 1993: Toward predictable biological control of Liriomyza trifolii (Diptera: Agromyzidae) infesting greenhouse cut chrysanthemums. Environ. Entomol. 22: 1217-1233.

HENDRICKSON R.M. JR. \& BARTH S.E. 1979: Introduced parasites of Agromyza frontella (Rondani) in the USA. J. New York Entomol. Soc. 87: 167-174.

Кıм J.H., Byoun Y.W., Kıм Y.H. \& Kıм H.Y. 2007: Ecological characteristics and storage condition of Diglyphus isaea (Walker) (Hymenoptera: Eulophidae), an ectoparasite of Liriomyza trifolii (Burgess) (Diptera: Agromyzidae). Korean J. Appl. Entomol. 46: 43-49.

LaSalle J. \& Parrella M.P. 1991: The chalcidoid parasites (Hymenoptera, Chalcidoidea) of economically important Liriomyza species (Diptera, Agromyzidae) in North America. Proc. Entomol. Soc. Wash. 93: 571-591.

Lei Z.R., Zhu C.J. \& Zhang C.Q. 2007: Risk analysis of alien invasive Liriomyza trifolii (Burgess) in China. Plant Prot. 33: 37-41 [in Chinese].

MEYhÖFER R. \& CASAS J. 1999: Vibratory stimuli in host location by parasitic wasps. J. Insect Physiol. 45: 967-971.

MeyhöFer R., Casas J. \& Dorn S. 1994: Host location by a parasitoid using leafminer vibrations: characterizing the vibrational signals produced by the leafmining host. Physiol. Entomol. 19: 349-359.
MinkenBerg O.P.J.M. 1989: Temperature effects on the life history of the eulophid wasp Diglyphus isaea, an ectoparasitoid of leafminers (Liriomyza spp.), on tomatoes. Ann. Appl. Biol. 115: 381-397.

Ode P.J. \& Heinz K.M. 2002: Host-size-dependent sex ratio theory and improving mass-reared parasitoid sex ratios. Biol. Control 24: 31-41.

Sher R.B., Parrella M.P. \& van Lenteren J.C. 1996: Integrated biological control of leafminers, Liriomyza trifolii, on greenhouse chrysanthemums. IOBC/WPRS Bull. 19: 147-150.

Valérie F.L., Bagnères A.G. \& Clément J.L. 1996: Role of plant volatiles in the search for a host by parasitoid Diglyphus isaea (Hymenoptera: Eulophidae). J. Chem. Ecol. 22: 541-558.

Viggiani G. 1964: La specializzazione entomoparassitica in alcuni Eulofidi (Hymenoptera, Chalcidoidea). Entomophaga 9: $111-118$.

Zhao Y.X. \& Kang L. 2002: The role of plant odors in the leafminer Liriomyza sativae (Diptera: Agromyzidae) and its parasitoid Diglyphus isaea (Hymenoptera: Eulophidae): orientation towards the host habitat. Eur. J. Entomol. 99: 445-450.

Zhu C.D., LaSalle J. \& Huang D.W. 2000: A review of the Chinese Diglyphus Walker (Hymenoptera: Eulophidae). Orient. Insects 34: 263-288.

Received August 19, 2010; revised and accepted February 15, 2012 